\title{
DETERMINACIÓN DE LAS TÉCNICAS EXISTENCIALES EN LOS ESTUDIANTES COMPROMETIDOS CON GRUPOS DE RESPONSABILIZACIÓN EN LA UNALM
}

\author{
Lic. Eduardo Flavio Ortiz Coronado \\ Profesor del Departamento de Ciencias Humanas de la \\ Universidad Nacional Agraria La Molina \\ edortiz@lamolina.edu.pe \\ Lic. Yvonne Iris Ruiz Zegarra \\ Profesora del Departamento de Ciencias Humanas de la \\ Universidad Nacional Agraria La Molina \\ vonny@lamolina.edu.pe
}

\begin{abstract}
Resumen
Esta investigación pretende comprender el fenómeno ético de la responsabilización entre los estudiantes de la UNALM para poder diseñar las estrategias que hagan sostenible esta reserva moral entre los jóvenes de La Molina. Para ello presenta en primer lugar, la evaluación cuantitativa en una muestra de dos grupos seleccionados entre estudiantes que pertenecen a "grupos de responsabilización" y estudiantes que no pertenecen a ellos, con el objetivo de comparar el comportamiento de los "factores existenciales" como temperamento genéticamente heredado, tipo de figuras paternas, mensajes emocionales y afectivos predominantes y ambiente o constelación simbólica, los cuales han generado ciertos "presupuestos existenciales", así como personalidades que tienden a comprometerse. En segundo lugar, presenta la evaluación cualitativa de algunas "técnicas existenciales" de narración autobiográfica intersubjetivamente practicada, las cuales han permitido desarrollar la capacidad de una persona adulta para afirmarse a sí mismos a través del autodescentramiento y la apertura a las otras historias personales y colectivas en solidaridad o en su defecto quedar atrapados en un proceso regresivo de sumisión a la autoridad externa.
\end{abstract}

Palabras claves: Personalidad, temperamento, comportamiento, técnicas existenciales.

\begin{abstract}
This research tries to comprehend the process of taking ethical responsibility among students of the UNALM. Furthermore it pretends to design strategies to sustain this given moral reserve in time. For this purpose it first presents a quantitative evaluation of two groups of students: one consists of those who belong to any of so called "associations of responsibility taking", the other consists of those who don't. We pretend to compare the dynamics of "existential factors" like temperamental traits as part of genetic heredity $(\mathrm{T})$, types of paternal figures $(\mathrm{P})$, predominant emotional and affective messages $(\mathrm{M})$, symbolic constellation of unconscious environment $(\mathrm{A})$ that generate "existential presuppositions" and ethically motivated personalities formed by them. In a second step, it shows a qualitative evaluation of some "existential techniques" of intersubjective autobiographic narrative which allowed the students to develop the capacity of an adult person to affirm themselves through de-centeredness and openness to other personal and collective biographies in solidarity, or else to get caught in a process of regression and submission to external authority.
\end{abstract}

Key words: Personality, temperament, behavior, existential techniques. 


\section{Introducción}

Este artículo tiene como trasfondo los objetivos que nos propusiéramos ya en el año 2001, a propósito del diseño inicial del curso interdisciplinario "Proyecto Universitario Personalizado" (fundamentos antropológicos para la ética), del Departamento de Ciencias Humanas, dirigido a los alumnos ingresantes de la UNALM de todas las carreras que aquí se ofrecen.

Planteábamos entonces, la necesidad de hacer una relectura de la historia personal en clave autobiográfica, para llegar a tener una mayor claridad respecto de la propia identidad personal, que se va logrando finalmente, como sostiene Buber, en el proceso de los intercambios con los otros significativos. Una vez conquistada esta conciencia, pretendíamos que sería más fácil trascender la mera obligación académica de aprobar cursos o especializarse en las técnicas de la carrera de manera individualista, así como la exigencia infantil que experimenta quien se ubica como receptor pasivo de la formación. Lo que perseguíamos en el fondo era formar el pensamiento para la conciencia crítica dirigida a la asunción de la propia responsabilidad hacia uno mismo y hacia los otros, mediante la generación de una idea y el diseño de un proyecto de vida adulta.

Como una consecuencia de esta tarea, la importancia de la presente investigación estriba en la intención de estudiar el fenómeno de la responsabilización entre los estudiantes de la UNALM para llegar a establecer las estrategias apropiadas en la facilitación de esta dinámica entre los es- tudiantes que no presentan una personalidad naturalmente comprometida, es decir, para poder replicarla en la misma universidad, así como en otros escenarios.

Otra razón que hace relevante este trabajo se refiere al contexto de carreras científico-técnicas que ofrece la UNALM, cuya naturaleza ha incentivado pocas investigaciones relacionadas a iniciativas de responsabilización de estudiantes en actividades que van más allá de lo académico y que promueven el desarrollo de capacidades para la participación en la vida universitaria (quizá el primer ejercicio de participación ciudadana), a pesar de existir no pocos grupos con este fin en la UNALM.

Los alcances de la presente investigación se circunscriben entre los estudiantes de la UNALM pertenecientes y no pertenecientes a grupos de responsabilización que van más allá de lo académico y que han sido formados por iniciativa de ellos mismos, con intereses variados y cuyas edades fluctúan entre 18 y 26 años.

La intención de este artículo se dirige a determinar de qué manera la confluencia de ciertos factores convergentes ha permitido la generación de "presupuestos existenciales" a modo de estructuras inconscientes de interpretación de la realidad y de "técnicas existenciales" que son mecanismos reactivos, también inconscientes ante el mundo percibido durante la infancia, pubertad y adolescencia, y de qué forma estas dos instancias han determinado la formación o no de cierto perfil de personalidad en la población evaluada. Los límites vienen marcados por la imposibilidad de objetivar dichos presupuestos o técnicas, dado que se llega a ellos sólo por inferencia. Sin em- 
bargo, esa inferencia no es gratuita, sino que la hemos derivado de la confluencia de los factores establecidos, a saber, el temperamento genéticamente heredado (T), el tipo de padres responsables de la formación (P), los mensajes emocionales y afectivos recibidos predominantemente (M) y el ambiente o constelación de símbolos que ha rodeado al sujeto durante su desarrollo (A). Con todo, no pretendemos hacer un análisis formulístico, sino tan sólo de probabilidades, a partir de los casos estudiados.

Como objetivos nos fijamos entonces, determinar los presupuestos existenciales y las técnicas existenciales en las que convergen los factores que intervienen en la formación de una identidad personal, como la que presentan los estudiantes que se responsabilizan por los otros a través de su pertenencia a algún grupo de responsabilización en La Molina. Además nos propusimos establecer las estrategias adecuadas para afianzar o desarrollar dichas técnicas existenciales y así poder motivar este mismo compromiso en aquellos que aún no han iniciado el proceso.

Antes de presentar los materiales y métodos de nuestra investigación, así como los resultados y la discusión respectiva, se hace necesario definir ciertos conceptos y enfoques que se involucran directamente con el tema de este artículo tales como, factores existenciales, confluencia $\mathrm{T}+\mathrm{P}+\mathrm{M}+\mathrm{A}$, presupuestos existenciales y técnicas existenciales.

\section{Presupuestos existenciales}

Cuando preguntamos por las capacidades morales, en nuestro caso por la responsa- bilización, nos topamos con un sujeto que posee capacidades y también límites. La pregunta moral, en última instancia y es la pregunta antropológica, ya que el portador de esta capacidad de responder no sólo por él mismo, sino también por los otros es un ser humano con sus propios condicionamientos y posibilidades para serlo. Ahora bien, si la ética personal ha venido gestándose a lo largo de la historia de cada individuo de manera particular y distinta, es porque cada cual ha pasado por un proceso de humanización también particular y distinto, donde es posible observar la formación de ciertos presupuestos existenciales, más allá de la conciencia de la persona.

Un presupuesto existencial es un supuesto o intuición simbólica, una manera particular de percibir sensiblemente los distintos aspectos de la realidad, que ha condicionado finalmente nuestra acción (praxis). Al mismo tiempo, nos han permitido existir con cierto sentido, por eso les llamaremos también "patrones de sentido". La historia de la formación de un presupuesto existencial es compleja, está condicionada por la confluencia de ciertos factores, a saber: la carga temperamental, la relación con nuestros progenitores, los mensajes emocionales y afectivos que hemos recibido y el ambiente en que nos hemos desarrollado.

\section{Factores existenciales o CONFLUENCIA T $+\mathbf{P}+\mathbf{M}+\mathbf{A}$}

Los factores existenciales considerados en nuestro enfoque son cuatro: el tipo de temperamento innato o carga biológica genéticamente heredada (T), el tipo 
de padres con los que nos hemos formado en el entorno familiar (P), los mensajes emocionales y afectivos que hemos recibido (M) y el ambiente o constelación de símbolos en el que nos hemos desarrollado (A). Los factores existenciales tienen una importante actividad fundacional durante la primera y segunda infancia, así como durante la pubertad y la adolescencia. Ellos son calificados como existenciales en la medida en que permiten al sujeto la existencia, porque como ser humano -a diferencia de otros-, está caracterizado por la conciencia. En este sentido, los factores existenciales son elementos que permiten construir las bases sobre las que se irá desarrollando la conciencia humana en el transcurso hacia la vida adulta. Nos referimos a estructuras o fundamentos que van facilitando la creación de presupuestos existenciales, o sea, una autoimagen, intuitiva primero, sin una clara certeza racional todavía, con una poderosa percepción simbólica del entorno y una vivencia emocional sensible, todo ello a causa del dinamismo interrelacional que va tejiendo la confluencia $\mathrm{T}+\mathrm{P}+\mathrm{M}+\mathrm{A}$. Ahora bien, la autoimagen preconceptual va formándose paralelamente a la construcción de la imagen de los otros en el seno familiar y más allá de él, los modelos y paradigmas culturales, el mundo físico, las cosmovisiones, creencias religiosas, y otros aspectos de la realidad. Todas estas percepciones son captadas a un nivel inconsciente, atemático y sensible, difuso y confuso, antes de producirse la tematización de la realidad, de manera clara y distinta en el futuro por operación de la conciencia.

\section{Temperamento innato y temperamento terapéutico $(\mathrm{T})$}

Cuando hablamos del temperamento, nos referimos a la carga biológica genéticamente heredada, o lotería biológica que nos ha tocado en la compleja estructura psico-físico-espiritual que nos sostiene. El temperamento está conformado por nuestro "yo esencial" o tendencia natural de acción y el "yo terapéutico" o resultado de la modificación a lo largo de la historia personal de estos impulsos o tendencias naturales. Cuando Riso hace esta distinción, se refiere a la totalidad de la personalidad en dos elementos constitutivos que la componen y que se reconfiguran mutuamente. Entonces, mientras va desarrollando el yo terapéutico o histórico (social) el yo esencial se adapta, pero sin desaparecer. La concientización de las propias reacciones emocionales e ideas permite ir haciendo esa adaptación. Por ejemplo, si tengo un yo esencial tendente a la depresión puedo aprender a reaccionar de una manera menos depresiva y más tendente a la euforia, para encontrar de esta forma un equilibrio entre las dos dimensiones de mi yo. Pero esto no significa que ante situaciones de crisis, toma de decisiones, adversidades, situaciones inesperadas o contrariedades, mi yo esencial no sea el primero en emerger, sólo que cada vez más adaptado a mis nuevas circunstancias, de adulto, más consciente, más racional y más dialógico.

Partiendo de Goleman, podemos decir que existen dos tipos de temperamento, que vamos a denominarlos, Tipo 1 y Tipo 2. Sin embargo, no todas las personas que nacen con T1 o T2 tienen la misma configuración de su temperamento, ya que cada una es un mundo amplio, complejo, insondable, irreductible a una fórmula que la determine o parametre. Las perso- 
nas están caracterizadas particularmente por unos rasgos esenciales que aparecen en cada una con propia configuración, es decir, con más fuerza y frecuencia que en otras, así como unos movimientos de intensidad y resonancia particulares frente a la realidad.

\begin{tabular}{|l|l|}
\hline \multicolumn{2}{|c|}{ Rasgos básicos del temperamento por tipos } \\
\hline \multicolumn{1}{|c|}{ TIPO 1 (T1) } & TIPO 2 (T2) \\
\hline Introversión & Extroversión \\
\hline Depresión & Euforia \\
\hline Tristeza & Alegría \\
\hline Temor & Valentía \\
\hline Cobardía & Riesgo \\
\hline Pasión & Acción \\
\hline Represión & Expresión \\
\hline Pesimismo & Optimismo \\
\hline Reflexión & Irreflexión \\
\hline Dependencia & Independencia \\
\hline Drama & Comedia \\
\hline Teoría & Praxis \\
\hline Conciencia & Inconsciencia \\
\hline Conservadurismo & Innovación \\
\hline Cerebro & Vísceras \\
\hline Fuerza endógena & Fuerza exógena \\
\hline Complejidad & Simplicidad \\
\hline Complicación & Resolución \\
\hline Formalidad & Informalidad \\
\hline Obsesión - Manía & $\begin{array}{l}\text { Ecuanimidad - } \\
\text { Serenidad }\end{array}$ \\
\hline Emoción & Sentimiento \\
\hline
\end{tabular}

\section{Tipo de padres que nos han criado (P)}

El tipo de padres con que nos hemos formado en el entorno familiar o en su lugar el modelo de padre y madre que hayamos tenido en las etapas de desarrollo inicial representan una influencia preponderante en nuestro desarrollo. Los indicadores son básicamente la edad cronológica sobre un nivel de madurez psicológica particular. Pues se puede ser un adulto a nivel cronológico y no haber desarrollado suficientemente como se espera de un adulto en niveles de responsabilización e independencias afectiva, económica o ideológica. Para llegar a comprender cómo se va dando la referida confluencia, hay que cruzar este segundo factor $(\mathrm{P})$ con el primero $(\mathrm{T})$. Por ejemplo, puedo haber crecido con un padre adulto que tiene un temperamento extrovertido y esta experiencia difiere de haberlo hecho con un padre adulto de temperamento introvertido, dígase lo mismo para el caso de la madre, incluso estos padres pueden o no haberlo modificado creando un temperamento más saludable. Ahora bien, cuando hablamos de tipo de padre, en realidad estamos hablando del primer referente de crianza, que puede ser el padre o madre biológicos, pero no con exclusividad, ya que también pudo haberme criado mi abuelo o mi tía o ambos y estos son en realidad mi Tipo de padre o mi paradigma de padre, con toda la fuerza determinante que este imprime.

Haber tenido un padre adulto o un padre niño ha influido en el desarrollo armonioso de la madurez psicológica de la persona, en el sentido de haber tenido o no un referente de orientación y seguridad fundamental. Un padre niño con reacciones emocionales no saludables, como diría Powell, es incapaz de reconocer su mundo psicoafectivo, negándolo o reprimiéndolo y con ello no haber favorecido la creciente capacidad adulta para afrontar los propios afectos. Un padre adulto, en cambio, favorece el desarrollo de un adulto, ya sea que la persona se encuentre en la niñez, la pubertad o la adolescencia. 


\section{Mensajes emocionales $y$ afectivos predominantes $(\mathbf{M})$}

En la configuración de la estructura humana, los mensajes emocionales y afectivos son determinantes. En realidad, no estamos diciendo que las personas han recibido ni únicamente mensajes positivos, ni únicamente negativos, pues todos hemos tenido, muy seguramente, la experiencia de ambos. Pero lo que sí queremos establecer es la necesidad de precisar el tipo de mensajes que ha predominado, pues, por ejemplo, será diferente la autoimagen, la autovaloración y las ideas que haya formado de sí mismo quien básicamente fue maltratado verbalmente, abusado en el cuerpo o burlado por algún defecto/característica peculiar, que quien recibió mensajes afirmativos. "No quiero volver a verte nunca más", "eres un niño muy malo", "qué feo eres", "siempre te equivocas", "estoy avergonzado de ser tu padre", son mensajes que contrastan con otros como "me siento muy bien a tu lado", "eres la persona más linda que he conocido en mi vida", "qué inteligente y hábil eres", "estamos orgullosos de ser tus padres". Estos mensajes van contribuyendo a la configuración de nuestra pauta existencial fundamental.

\section{Ambiente de desarrollo o constelación de símbolos (A)}

El factor ambiente es otro de los factores fundamentales de la confluencia. El ambiente se define como la constelación de símbolos que han rodeado al sujeto a lo largo de su desarrollo. Los símbolos no son precisamente mensajes tan explícitos como los conceptos o las ideas, pero sí tie- nen una fuerza mayor en el inconsciente del individuo, que subyace a ese nivel influyendo de manera activa en la dinámica personal. Símbolos pueden ser los colores que nos han rodeado, la luz u oscuridad de los espacios que hemos habitado, la cercanía a la naturaleza o la ciudad en la infancia y pubertad, el tono en la voz de las personas que nos criaron o cuidaron, las actitudes de apertura o cerrazón de los padres y las otras personas con quienes tuvimos contacto, la exposición o sobreprotección en el proceso de socialización, el temperamento mismo de los padres o personas mayores del entorno, los gestos faciales, las maneras de incluir la corporeidad en la experiencia humana, percibidos incluso desde la vida intrauterina.

Ahora bien, respecto de (M) y de (A), hay que decir que son vitales, dada la estructura previa de la conciencia humana en formación, la cual capta las ideas emocionales como también los símbolos emocionales y los capta como intuiciones que los otros o la realidad misma les van comunicando.

\section{Técnicas existenciales desarrolladas a partir de los presupuestos formados}

Las técnicas existenciales son la forma de funcionamiento existencial o la manera de afrontar la vida que nuestros presupuestos existenciales nos han permitido operar ante la realidad que se nos imponía y nos afectaba con sus determinaciones. Dicho de otro modo, las técnicas existenciales son como mecanismos de reacción previsible ante las situaciones diversas que vamos experimentando y que provocan de nues- 
tra parte una respuesta concreta, a partir de nuestros presupuestos o presuposiciones condicionantes frente a la vida.

La relación que existe entre los presupuestos existenciales y las técnicas existenciales es de una proporción directa, esto es que, un presupuesto existencial es la dimensión "predisposicional", pues la confluencia $\mathrm{T}+\mathrm{P}+\mathrm{M}+\mathrm{A}$ nos ha proveído de ciertas suposiciones o predisposiciones, así como de una actitud característica para participar en el mundo de manera particular. La técnica existencial en cambio es la dimensión "actual u operacional” con que actúo o me comporto, ya en sentido práctico frente a un estímulo de la realidad.

Sin embargo, tanto la dimensión predisposicional como la operacional permanecen en el ámbito humano del inconsciente. Son movimientos que normalmente no han sido ni son hecho conscientes, pues aunque los poseemos como disposiciones y mecanismos de funcionamiento no nos percatamos normalmente de su dinamismo. Se hace, por supuesto, necesario un discernimiento existencial, es decir de tematización consciente de las intuiciones y clarificación de estos símbolos y sensaciones (presupuestos y técnicas existenciales), los cuales una vez trascendidos nos permitirán construir argumentos para una existencia humana más integral.

\section{3. "Esther": caso paradigmático}

Un ejemplo que podría graficar los conceptos y dinámicas que hemos venido describiendo es el caso de "Esther". Una niña que a los 5 ańos ha perdido a su madre de temperamento T2. Ella es una niña de un temperamento $\mathrm{T} 1$ que ha quedado al cuidado de un Padre niño, quien además es un $\mathrm{T} 1$ sin temperamento terapéutico trabajado. Este ha llevado a la abuela a casa para encargarla de la formación de la niña, pero esta mujer ha comunicado en su relación con Esther lo mismo que él, o sea mensajes emocionales y afectivos de tristeza, desconfianza y desánimo: "Tienes que guardar, por lo menos 5 años más de luto, porque tu madre se lo merece", le ha dicho la abuela cuando a los 14 años ella le manifestara su deseo de celebrar el quinceañero. Pero además, desde la muerte de la madre, Esther sólo ha escuchado de su padre mensajes como: "Ay hijita, hemos perdido la razón de nuestra vida, se ha muerto el pilar de nuestro hogar, nunca volveremos a ser felices". Respecto del Ambiente o constelación de símbolos que ha rodeado a Esther tenemos que, creció en una "casa cerrada", sin más familia ni primos, ni tíos que compartan la vida con sus cotidianas alegrías y penas, ni amigos y amigas que visiten; el juego no ha sido permitido al interior de la casa, no ha habido paseos de esparcimiento familiar o social, las cortinas negras han permanecido cerradas siempre junto con las ventanas por temor a que el viento traiga enfermedades; el cuarto de la madre muerta nunca ha sido abierto ni utilizado por otra persona nuevamente; un hogar silente y quejoso, con lágrimas, caras de luto y de dolor, sin expresión franca de las emociones y con respetos exagerados hacia los mayores o hacia las situaciones individuales que cada miembro iba viviendo.

Estos son los factores existenciales que han acompañado el desarrollo de Esther y que 
han creado en ella algunos presupuestos existenciales o presuposiciones condicionantes, como por ejemplo, la intuición de que es "una huérfana", la autoimagen débil y poco afirmada de sí misma, la sensación de que necesita recibir cuidado y protección constantes, el supuesto de que ella no puede prodigarlos a otros, la intuición no conceptualizada de que necesita a alguien más fuerte que ella para poder hacer frente a las adversidades, la convicción no tematizada de que ella no es una adulta y por lo tanto será incapaz de convertirse en una madre engendradora de vida para otros, etc.

Ahora bien, como hemos dicho, los presupuestos existenciales generan a su vez técnicas existenciales en las personas, o mecanismos de reacción operacional. En el caso planteado serán la timidez y pasividad para sostener una idea propia en debates o controversias, el reclamo infantil de cuidado y protección constantes frente a un peligro o situación de dificultad, el desinterés de generar ideas para otros o para sí misma ante una necesidad detectada, la sumisión o sometimiento a la autoridad en vez de la toma de decisiones independientemente; la dependencia (afectiva, física, ideológica o material) el comportamiento infantil con los adultos y la manipulación de sus relaciones, la indefinición de un proyecto de vida adulta, la dramatización habitual o sobredimensionamiento de las situaciones o problemas cotidianos de normal tratamiento, la sensibilidad excesiva o susceptibilidad frente a eventos neutros, el temor intenso a la soledad, el poco control de la tristeza ante situaciones de pérdida o muerte.

\section{MATERIALES Y MÉTODOS}

El primer instrumento utilizado en la presente investigación es la Encuesta $\mathbf{T}+\mathbf{P}+\mathbf{M}+\mathrm{A}$ elaborada por los autores de este artículo, docentes del Departamento de Ciencias Humanas de la Universidad Nacional Agraria La Molina; la cual consta de 40 preguntas dirigidas a caracterizar los factores existenciales que intervienen en la formación de los presupuestos existenciales y estos a su vez, en la formación de las técnicas o mecanismos existenciales. Las preguntas acerca de cada factor fueron intercaladas a lo largo de la encuesta. Después de elaborarla la sometimos a la validación por parte de los jueces expertos en el tema (profesionales en ciencias sociales, psicología y filosofía) y en el caso de los 20 alumnos jueces de la UNALM, a través del método de comprensión lectora. Ambos grupos aportaron de manera significativa a la reelaboración definitiva de la encuesta.

Finalmente, procedimos a la evaluación de una población de 74 jóvenes estudiantes de la UNALM, teniendo en cuenta las variables de sexo, edad, carrera profesional, grupo de pertenencia, nivel socioeconómico, lugar de nacimiento, instrucción de los padres, y motivos de pertenencia al grupo, entre los más importantes. Se aplicó a 30 hombres y 44 mujeres, cuyas edades promedio están comprendidas entre 18 y 26 años, de todas las carreras de la UNALM y de los grupos de responsabilización Los encuestados fueron 56 limeños y 18 provincianos.

Para la presente investigación se utilizó 
la técnica de selección simple, buscando que fuera una muestra representativa de la población de alumnos que pertenecen y que no pertenecen a grupos de responsabilización de la UNALM, con el fin de establecer las diferencias. Además, la muestra fue tomada de manera noproporcional en referencia a la cantidad de estudiantes por facultad. La forma de aplicación de la encuesta fue a través de convocatorias a los grupos de interés por separado y su llenado de manera individual, explicándoles el objetivo de estudiar el fenómeno del compromiso más allá de lo académico, entre los estudiantes de la UNALM que conforman dichos grupos, para desarrollar las características descubiertas en la población general de la universidad y diseñar las estrategias del caso, en los cursos - preferentemente del Departamento de Ciencias Humanas- que tienen una orientación hacia el desarrollo personal y la responsabilización social.

El segundo instrumento empleado fue la entrevista personal con el fin de individualizar los resultados entre la población encuestada y establecer las diferencias de manera cualitativa. El primer paso consistió en mostrar individualmente los resultados de la propia encuesta a cada interesado, con el objeto de hacer una exploración inicial de los niveles de conciencia acerca de sus propias respuestas, es decir, el comportamiento de los factores existenciales en su propia historia personal. Asimismo, este ejercicio se dirigió a facilitar la identificación de los posibles presupuestos existenciales generados por la confluencia $\mathrm{T}+\mathrm{P}+\mathrm{M}+\mathrm{A}$ y sus consecuentes técnicas o mecanismos existenciales. En cada entrevista entregamos los resultados separados por factor a cada participante, con el fin de ayudar a la visualización y ponderación de los mismos. La intención de fondo era provocar reacciones en ellos, recoger sus impresiones y generar un diálogo a partir de esas percepciones subjetivas para conocer el alcance más integral de sus respuestas. Además, establecimos el criterio de facilidad de verbalización o narración de los procesos personales de la propia biografía para ser medido cualitativamente durante la entrevista personal.

\section{RESULTADOS Y DISCUSIÓN}

CUADRO 1: Distribución porcentual de motivos de participación en el grupo de referencia

\begin{tabular}{|c|c|c|c|c|c|c|c|}
\hline & \multirow[t]{2}{*}{ Total } & \multicolumn{2}{|c|}{ Sexo } & \multicolumn{3}{|c|}{ Edad } & $\begin{array}{l}\text { Grupo de } \\
\text { Referencia }\end{array}$ \\
\hline & & Hombres & Mujeres & Hasta 20 & 21 a 23 & 24 a mas & Con Grupo \\
\hline BASES ABSOLUTAS & $(29)$ & (10) & (19) & (9) & (14) & (6) & (29) \\
\hline Amistades lo invitaron & 27.6 & 20.0 & 31.6 & 33.3 & 14.3 & 50.0 & 27.6 \\
\hline $\begin{array}{l}\text { Quería hacer algo por } \\
\text { los demás }\end{array}$ & 17.2 & 20.0 & 15.8 & 44.4 & 7.1 & & 17.2 \\
\hline Quería sentirme útil & 13.8 & 20.0 & 10.5 & & 21.4 & 16.7 & 13.8 \\
\hline $\begin{array}{l}\text { Hay que tener una } \\
\text { actividad social o de } \\
\text { liderazgo }\end{array}$ & 41.4 & 40.0 & 42.1 & 22.2 & 57.1 & 33.3 & 41.4 \\
\hline
\end{tabular}


ESTADIGRAFO: \% COLUMNA

Fuente: Elaboración propia

El primer resultado se refiere al motivo de participación principal en el grupo de referencia, encontrando el significativo porcentaje de $\mathbf{5 8 . 6} \%$ que refiere razones de convicción y compromiso social. Este porcentaje está conformado por el $41.4 \%$ que afirman su convicción acerca de la necesidad de "tener una actividad social o de liderazgo en su vida" y por el $17.2 \%$ que desea "hacer algo por los demás", en lugar de tener simplemente razones individualistas como "sentirse útil" o responder a una invitación externa sin iniciativa directa de compromiso social.

El cuadro 2 permite observar una diferencia porcentual del $14.2 \%$ favorable a la toma de decisiones en los encuestados que pertenecen a grupos de referencia, en contraste de aquellos que no pertenecen a ninguno. Esto supone que las personas con características temperamentales de resuelta decisión no se entrampan en su dinámica personal, sino que han podido trascenderse a sí mismos y dar un aporte significativo en sus compromisos con grupos de referencia $(58.6 \%$ frente al 44.4\%).

Del 100\% de encuestados el 69\% de los que pertenecen a un grupo de referencia ha tenido la experiencia de unos padres emocionalmente inteligentes que le han permitido la franca expresión de su mundo emocional y afectivo, versus un $42.3 \%$ que representa a quienes no pertenecen, notando en este último grupo la predominancia hacia una menor oportunidad para haber expresado los afectos. Obsérvese la gran diferencia de $26.7 \%$, lo cual permite afirmar la importancia fundamental de la integración psíquica de la condición humana, que funda capacidades o las castra sin dejar lugar para su despliegue.

De la población de quienes pertenecen a un grupo de referencia, el $27.6 \%$ ha tenido unos padres que no se han comprometido activamente con la realidad sino que se han entregado normalmente a la construcción de grandes ideales, mientras que ese mismo fenómeno es observable en un $42.2 \%$ en la población de los que no conforman un grupo de referencia, sólo que comparativamente en una medida más considerable (14.6\%). Este resultado nos llama la atención pues sería de esperar que quienes están comprometidos en grupos hubieran tenido padres comprometidos como resultado de un gran idealismo y quienes no integran grupos más bien hubieran tenido padres menos idealistas que los del primer grupo y al mismo tiempo no comprometidos. Sin embargo, podemos deducir de esta información que, por un lado, la construcción de los ideales no siempre supone un compromiso militante con la realidad y por otro, que muchas veces, más bien, la experiencia de haber tenido padres con una praxis de compromiso y no sólo con grandes ideales es lo que genera consecuentemente un compromiso en las personas.

En relación a los mensajes positivos por los logros alcanzados el cuadro 5 muestra que el $68.9 \%$ de aquellos que sí pertenecen a un grupo de referencia los recibió en gran medida, mientras que sólo el $51.1 \%$ de quienes no pertenecen a estos grupos recibió también este tipo de mensajes. Una diferencia del $17.8 \%$ es un indicador 
CUADRO 2: Distribución porcentual de concordancia con la frase: "Soy de los que toma decisiones resueltamente, más que de los que se complican”

\begin{tabular}{|l|c|c|c|c|c|c|c|c|}
\hline \multirow{2}{*}{} & \multirow{2}{*}{ Total } & \multicolumn{2}{|c|}{ Sexo } & \multicolumn{3}{c|}{ Edad } & \multicolumn{2}{c|}{$\begin{array}{c}\text { Grupo de } \\
\text { referencia }\end{array}$} \\
\cline { 3 - 9 } & & Hombres & Mujeres & $\begin{array}{c}\text { Hasta } \\
20\end{array}$ & 21 a 23 & 24 a más & $\begin{array}{c}\text { Con } \\
\text { grupo }\end{array}$ & $\begin{array}{c}\text { Sin } \\
\text { grupo }\end{array}$ \\
\hline $\begin{array}{l}\text { Bases } \\
\text { Absolutas }\end{array}$ & $(74)$ & $(30)$ & $(44)$ & $(18)$ & $(40)$ & $(16)$ & $(29)$ & $(45)$ \\
\hline $\begin{array}{l}1 . \text { Totalmente } \\
\text { en desacuerdo }\end{array}$ & 4.1 & & 6.8 & & 5.0 & 6.3 & & 6.7 \\
\hline 2 & 8.1 & 3.3 & 11.4 & 5.6 & 12.5 & & 6.9 & 8.9 \\
\hline 3 & 37.8 & 40.0 & 36.4 & 38.9 & 35.0 & 43.8 & 34.5 & 40.0 \\
\hline $\mathbf{4}$ & 31.1 & 26.7 & 34.1 & 44.4 & 30.0 & 18.8 & $\mathbf{3 1 . 0}$ & $\mathbf{3 1 . 1}$ \\
\hline $\begin{array}{l}\mathbf{5} \text { 5. Totalmente } \\
\text { de acuerdo }\end{array}$ & 18.9 & 30.0 & 11.4 & 11.1 & 17.5 & 31.3 & $\mathbf{2 7 . 6}$ & $\mathbf{1 3 . 3}$ \\
\hline $\begin{array}{l}\text { ESTADIGRAFO: \% COLUMNA } \\
\text { Fuente: Elaboración propia }\end{array}$
\end{tabular}

CUADRO 3: Distribución porcentual de concordancia con la frase: "Mi infancia y pubertad las he pasado aprendiendo de mis padres la expresión de los afectos y emociones, más que el control represivo de los mismos"

\begin{tabular}{|c|c|c|c|c|c|c|c|c|}
\hline & \multirow{2}{*}{ Total } & \multicolumn{2}{|c|}{ Sexo } & \multicolumn{3}{|c|}{ Edad } & \multicolumn{2}{|c|}{$\begin{array}{l}\text { Grupo de } \\
\text { referencia }\end{array}$} \\
\hline & & Hombres & Mujeres & $\begin{array}{c}\text { Hasta } \\
20\end{array}$ & 21 a 23 & 24 a máss & $\begin{array}{l}\text { Con } \\
\text { grupo }\end{array}$ & $\begin{array}{c}\text { Sin } \\
\text { grupo }\end{array}$ \\
\hline Bases absolutas & $(74)$ & (30) & $(44)$ & (18) & $(40)$ & (16) & (29) & $(45)$ \\
\hline $\begin{array}{l}\text { 1. Totalmente } \\
\text { en desacuerdo }\end{array}$ & 2.7 & 3.3 & 2.3 & & 2.5 & 6.3 & 3.4 & 2.2 \\
\hline 2 & 13.5 & 10.0 & 15.9 & & 20.0 & 12.5 & 6.9 & 17.8 \\
\hline 3 & 29.7 & 36.7 & 25.0 & 16.7 & 37.5 & 25.0 & 20.7 & 35.6 \\
\hline 4 & 32.4 & 40.0 & 27.3 & 44.4 & 25.0 & 37.5 & 41.4 & 26.7 \\
\hline $\begin{array}{l}\text { 5. Totalmente } \\
\text { de acuerdo } \\
\end{array}$ & 20.3 & 10.0 & 27.3 & 38.9 & 12.5 & 18.8 & 27.6 & 15.6 \\
\hline No responde & 1.4 & & 2.3 & & 2.5 & & & 2.2 \\
\hline
\end{tabular}

elevado, de lo cual inferimos que la afirmación psicoafectiva que reciben las personas durante su desarrollo es una experiencia fundamental de -tal como su nombre lo sugiere- la capacidad de quedar afectado por la vulnerabilidad humana que lo rodea, la presencia y carencia del otro, para comprometerse y responsabilizarse.

Según el cuadro No 6 podemos observar que la presencia y cercanía ante situaciones de dificultad y, en general, los procesos personales experimentados durante la historia de quienes participan en un grupo 
CUADRO 4: Distribución porcentual de concordancia con la frase: "Mis padres se han entregado normalmente a la construcción de un gran sueńo o ideal, antes que al ejercicio activo de compromisos por la realidad"

\begin{tabular}{|l|c|c|c|c|c|c|c|c|}
\hline \multirow{2}{*}{} & \multirow{2}{*}{} & \multicolumn{2}{|c|}{ Sexo } & \multicolumn{4}{c|}{ Edad } & \multicolumn{2}{c|}{$\begin{array}{c}\text { Grupo de } \\
\text { referencia }\end{array}$} \\
\cline { 2 - 10 } & Total & Hombres & Mujeres & $\begin{array}{c}\text { Hasta } \\
20\end{array}$ & $21 \mathrm{a} 23$ & 24 a más & $\begin{array}{c}\text { Con } \\
\text { grupo }\end{array}$ & $\begin{array}{c}\text { Sin } \\
\text { grupo }\end{array}$ \\
\hline Bases absolutas & $(74)$ & $(30)$ & $(44)$ & $(18)$ & $(40)$ & $(16)$ & $(29)$ & $(45)$ \\
\hline $\begin{array}{l}1 . \text { Totalmente } \\
\text { en desacuerdo }\end{array}$ & 5.4 & & 9.1 & 5.6 & 5.0 & 6.3 & 6.9 & 4.4 \\
\hline 2 & 20.3 & 20.0 & 20.5 & 22.2 & 25.0 & 6.3 & 24.1 & 17.8 \\
\hline 3 & 35.1 & 33.3 & 36.4 & 27.8 & 37.5 & 37.5 & 37.9 & 33.3 \\
\hline $\mathbf{4}$ & 23.0 & 26.7 & 20.5 & 27.8 & 22.5 & 18.8 & $\mathbf{6 . 9}$ & $\mathbf{3 3 . 3}$ \\
\hline $\begin{array}{l}5 . \text { Totalmente } \\
\text { de acuerdo }\end{array}$ & 13.5 & 16.7 & 11.4 & 16.7 & 5.0 & 31.3 & $\mathbf{2 0 . 7}$ & $\mathbf{8 . 9}$ \\
\hline \begin{tabular}{l} 
No responde \\
\hline
\end{tabular} & 2.7 & 3.3 & 2.3 & & 5.0 & & 3.4 & 2.2 \\
\hline $\begin{array}{l}\text { ESTADIGRAFO: \% COLUMNA } \\
\text { Fuente: Elaboración propia }\end{array}$
\end{tabular}

CUADRO 5: Distribución porcentual de concordancia con la frase: "En mi infancia he recibido fundamentalmente mensajes de felicitación por los logros, más que indiferencia por las pequeñas metas alcanzadas"

\begin{tabular}{|l|c|c|c|c|c|c|c|c|}
\hline \multirow{2}{*}{} & \multirow{2}{*}{} & \multicolumn{2}{|c|}{ Sexo } & \multicolumn{3}{c|}{ Edad } & \multicolumn{2}{c|}{ Grupo de referencia } \\
\cline { 3 - 10 } & Total & Hombres & Mujeres & $\begin{array}{c}\text { Hasta } \\
20\end{array}$ & 21 a 23 & $\begin{array}{c}24 \text { a } \\
\text { más }\end{array}$ & $\begin{array}{c}\text { Con } \\
\text { grupo }\end{array}$ & $\begin{array}{c}\text { Sin } \\
\text { grupo }\end{array}$ \\
\hline Bases absolutas & $(74)$ & $(30)$ & $(44)$ & $(18)$ & $(40)$ & $(16)$ & $(29)$ & $(45)$ \\
\hline $\begin{array}{l}1 . \text { Totalmente } \\
\text { en desacuerdo }\end{array}$ & 5.4 & 3.3 & 6.8 & & 7.5 & 6.3 & 3.4 & 6.7 \\
\hline 2 & 12.2 & 6.7 & 15.9 & 5.6 & 12.5 & 18.8 & 10.3 & 13.3 \\
\hline 3 & 23.0 & 30.0 & 18.2 & 11.1 & 27.5 & 25.0 & 17.2 & 26.7 \\
\hline $\mathbf{4}$ & 33.8 & 43.3 & 27.3 & 44.4 & 32.5 & 25.0 & $\mathbf{3 7 . 9}$ & $\mathbf{3 1 . 1}$ \\
\hline $\begin{array}{l}\text { 5. Totalmente } \\
\text { de acuerdo }\end{array}$ & 24.3 & 16.7 & 29.5 & 38.9 & 17.5 & 25.0 & $\mathbf{3 1 . 0}$ & $\mathbf{2 0 . 0}$ \\
\hline \begin{tabular}{l} 
No responde \\
\hline
\end{tabular} & 1.4 & & 2.3 & & 2.5 & & & 2.2 \\
\hline $\begin{array}{l}\text { ESTADIGRAFO: \% COLUMNA } \\
\text { Fuente: Elaboración propia }\end{array}$
\end{tabular}


CUADRO 6: Distribución porcentual de concordancia con la frase: "Normalmente me daban mensajes que me hacían sentir la cercanía y atención a mis problemas y a mis procesos personales, más que indiferencia frente a ellos"

\begin{tabular}{|c|c|c|c|c|c|c|c|c|}
\hline & \multirow[b]{2}{*}{ Total } & \multicolumn{2}{|c|}{ Sexo } & \multicolumn{3}{|c|}{ Edad } & \multicolumn{2}{|c|}{ Grupo de referencia } \\
\hline & & Hombres & Mujeres & Hasta 20 & 21 a 23 & $\begin{array}{l}24 \text { a } \\
\text { más }\end{array}$ & $\begin{array}{l}\text { Con } \\
\text { grupo }\end{array}$ & Sin grupo \\
\hline $\begin{array}{l}\text { Bases } \\
\text { absolutas }\end{array}$ & $(74)$ & (30) & $(44)$ & (18) & $(40)$ & $(16)$ & (29) & $(45)$ \\
\hline $\begin{array}{l}\text { 1. Totalmente } \\
\text { en desacuerdo }\end{array}$ & 4.1 & & 6.8 & & 5.0 & 6.3 & & 6.7 \\
\hline 2 & 5.4 & 6.7 & 4.5 & & 7.5 & 6.3 & 3.4 & 6.7 \\
\hline 3 & 27.0 & 23.3 & 29.5 & 22.2 & 30.0 & 25.0 & 27.6 & 26.7 \\
\hline 4 & 48.6 & 56.7 & 43.2 & 55.6 & 45.0 & 50.0 & 55.2 & 44.4 \\
\hline $\begin{array}{l}\text { 5. Totalmente } \\
\text { de acuerdo }\end{array}$ & 14.9 & 13.3 & 15.9 & 22.2 & 12.5 & 12.5 & 13.8 & 15.6 \\
\hline
\end{tabular}

CUADRO 7: Distribución porcentual de concordancia con la frase: "En el barrio en el que crecí predominaba la colectividad y el compromiso, antes que el individualismo egoísta y competitivo y el desinterés de unos hacia otros"

\begin{tabular}{|l|c|c|c|c|c|c|c|c|}
\hline & & \multicolumn{2}{|c|}{ Sexo } & \multicolumn{3}{c|}{ Edad } & \multicolumn{2}{c|}{ Grupo de referencia } \\
\cline { 3 - 9 } & Total & Hombres & Mujeres & $\begin{array}{c}\text { Hasta } \\
20\end{array}$ & 21 a 23 & $\begin{array}{c}24 \text { a } \\
\text { más }\end{array}$ & $\begin{array}{c}\text { Con } \\
\text { grupo }\end{array}$ & $\begin{array}{c}\text { Sin } \\
\text { grupo }\end{array}$ \\
\hline $\begin{array}{l}\text { Bases } \\
\text { absolutas }\end{array}$ & $(74)$ & $(30)$ & $(44)$ & $(18)$ & $(40)$ & $(16)$ & $(29)$ & $(45)$ \\
\hline $\begin{array}{l}1 . \\
\text { Totalmente } \\
\text { en } \\
\text { desacuerdo }\end{array}$ & 12.2 & 10.0 & 13.6 & 5.6 & 12.5 & 18.8 & 17.2 & 8.9 \\
\hline 2 & 16.2 & 6.7 & 22.7 & 5.6 & 25.0 & 6.3 & 10.3 & 20.0 \\
\hline 3 & 32.4 & 43.3 & 25.0 & 38.9 & 25.0 & 43.8 & 27.6 & 35.6 \\
\hline $\mathbf{4}$ & 17.6 & 10.0 & 22.7 & 16.7 & 22.5 & 6.3 & $\mathbf{2 4 . 1}$ & $\mathbf{1 3 . 3}$ \\
\hline $\begin{array}{l}\mathbf{5 .} \\
\text { Totalmente } \\
\text { de acuerdo }\end{array}$ & 14.9 & 20.0 & 11.4 & 22.2 & 10.0 & 18.8 & $\mathbf{1 3 . 8}$ & $\mathbf{1 5 . 6}$ \\
\hline No responde & 6.8 & 10.0 & 4.5 & 11.1 & 5.0 & 6.3 & 6.9 & 6.7 \\
\hline
\end{tabular}

ESTADIGRAFO: \% COLUMNA

Fuente: Elaboración propia 
de referencia, tiene un 69\% de incidencia, mientras que en quienes no pertenecen a tales grupos, la experiencia se da en menor medida, el 60\%. Ello demuestra una capacidad gestada gracias a la presencia de unos padres atentos al mundo interior y relacional de sus hijos y a los aspectos de éxito y fracaso afrontados con solicitud pertinente. Una capacidad para estar atentos ellos también, ahora que son adultos a los procesos de los otros, en mayor medida que la que puede constatarse entre quienes no pertenecen a grupos de responsabilización.

En este gráfico se muestra que los encuestados que pertenecen a grupos de responsabilización crecieron en un ambiente mediato o barrio en el que predominaba la colectividad y el compromiso, mientras que quienes no pertenecen a un grupo de referencia les sucedió en menor medida. Ello demuestra que el contexto social primario de pertenencia a subgrupos como un vecindario tiene una influencia poderosa en la manera de responsabilizarse. La persona va erigiéndose tal en un medio social que lo configura con características o no de responsabilización social.

\section{Resultados de las entrevistas perso- nales}

Los resultados de las entrevistas representan la medición cualitativa de las técnicas existenciales en los estudiantes de la UNALM que pertenecen a grupos de res-ponsabilización, encontrando que todos los entrevistados de este grupo - a diferencia de quienes no están comprometidos-, presentan niveles de conciencia bastante más profundos acerca de la dinámica de su historia personal, con referencia al comportamiento de la confluencia $\mathrm{T}+\mathrm{P}+\mathrm{M}+\mathrm{A}$.

Durante las entrevistas fue notoria la capacidad para narrarse que poseen en mayor medida, quienes pertenecen a diferencia de quienes no pertenecen a un grupo de referencia. En este sentido, su facilidad para afrontar incluso lo menos grato de su autobiografía permitió realizar con éxito, en esa misma sesión de la entrevista, el ensayo de ubicar e identificar sus propios presupuestos existenciales. Evidente fue también la diferencia cuando la totalidad de los estudiantes del primer grupo mostró lenguaje diferente de conceptos, enfoques y términos más precisos para poder narrarse, lo cual no hizo el grupo de los estudiantes del segundo grupo.

Observamos que quienes están comprometidos mostraron haber realizado alguna vez un ejercicio narrativo intersubjetivo de su vida, lo cual les permitió observarla desde otras perspectivas y dotarla de significados nuevos. La totalidad de los entrevistados refirieron que en algunas ocasiones durante su vida han realizado intercambios intersubjetivos con más de uno de los siguientes instrumentos, indicados por ellos mismos: 1) conversaciones periódicas con un consejero o acompañante personal o un profesional afín, y sobre todo con un amigo incondicional; 2) participación en talleres de desarrollo personal cuyas dinámicas consistían en compartir vivencias personales para luego analizarlas a la luz de nuevos marcos interpretativos; 3) práctica regular o espaciada de escribir un diario personal; 4) ejercicio de escribir la autobiografía en el curso de Proyecto Universitario Personalizado o la historia del "desarrollo personal -clave Fromm-Fowler” en 
el curso de Ética.

\section{Conclusiones}

1. Los estudiantes que pertenecen a grupos de responsabilización lo hacen notablemente movidos por convicciones personales de compromiso social, presentando temperamentos decididos y resueltos.

2. El perfil de personalidad de quienes pertenecen a grupos de responsabilización es el de aquellos que han tenido padres emocionalmente inteligentes que les facilitaron un clima expresivo de las emociones y atención a sus afectos, lo cual generó en ellos una especial atención y sensibilidad para dejarse afectar por la vulnerabilidad de las otras personas y por los problemas sociales que les aquejan.

3. Los estudiantes que forman parte de un grupo de referencia no han crecido con padres que hayan tenido precisamente una praxis de compromiso de responsabilización, aunque sí unos importantes ideales sociales de los cuales han aprendido sus hijos.

4. Los estudiantes que pertenecen a un grupo que va más allá de lo académico en la UNALM han crecido en subgrupos donde predominaba la colectividad y el compromiso, lo cual motiva en el presente su responsabilización en los nuevos subgrupos o contextos de acción donde se comprometen.

5. La medición cuantitativa de las características de los estudiantes que perte- necen y los estudiantes que no pertenecen a grupos de responsabilización en la UNALM, no muestra diferencias significativas, frente a la medición cualitativa que sí lo hace notablemente, concluyendo que es menos importante el contenido de lo vivido en la historia personal cuanto más las oportunidades para comprender el propio desarrollo personal e integrar de esta manera los aspectos positivos y los aparentemente negativos, en el conjunto de la experiencia total del sujeto.

6. La gran mayoría de los entrevistados que pertenecen a un grupo de responsabilización en la UNALM presentan como característica potencial unas técnicas o mecanismos existenciales de intercambio dialógico e intersubjetivo (no sólo individual) que les permiten reinterpretar y resignificar los acontecimientos de su propia vida.

7. Los estudiantes molineros que se comprometen en un grupo de responsabilización lo hacen porque las técnicas existenciales de narración autobiográfica intersubjetivamente practicada les han permitido desarrollar la capacidad adulta de autodescentramiento o toma de distancia de ellos mismos para poder dedicarse y solidarizarse con otras historias y problemas personales y colectivos, que no únicamente los suyos. Esto, justamente cuando aún están en camino de resolverse y comprenderse de una manera más integral.

8. El discernimiento de los presupuestos existenciales y las técnicas existenciales es un ejercicio impostergable que 
recomendamos implementar a través de los cursos de desarrollo personal para poder comprender y promover el fenómeno de su responsabilización ético-social entre los estudiantes de la UNALM

\section{Bibliografía}

DIETRICH, Georg. Psicología General del Counseling. Teoría y Práctica. España, Editorial Herder, 1986.

RISO, Richard. Describe tu perfil de personalidad en el eneagrama. Espańa, Editorial Desclée De Brouwer, 1997.
GOLEMAN, Daniel. La inteligencia emocional, porqué es más importante que el cociente intelectual. Argentina, Javier Vergara Editores, 1998.

FRANKL, Viktor. El hombre en busca de sentido. España, Editorial Herder, 1991.

POWELL, John. Plenamente humano, plenamente vivo. Una nueva vida a través de una nueva visión. España, Sal Terrae, 1998.

MUSSCHENGA, Albert W. La identidad personal y la sociedad individualizada. En: CONCILIUM, Revista Internacional de Teología, abril 2000, no 285, 2000. 27 - 36 pp. 


\section{ANEXO - ENCUESTA}

UNIVERSIDAD NACIONAL AGRARIA LA MOLINA

Departamento Académico de Ciencias Humanas

Prof. Eduardo Ortiz Coronado

Prof. Ivonne Ruiz Zegarra

\section{ENCUESTA $\mathrm{T}+\mathrm{P}+\mathrm{M}+\mathrm{A}$}

( ) Alumno/a

( ) Egresado/a

Grupo de referencia Tiempo de pertenencia

Carrera Ciclo

Edad Sexo

Lugar de nacimiento Distrito de Residencia

Núm. Hermanos

¿Con quiénes vives? (nombra todas las personas de tu casa):

Grado de instrucción, profesión y ocupación de papá:

Grado de instrucción, profesión y ocupación de mamá:

¿Con qué servicios de salud cuentan tus padres?

Pública ()Privada () Otros ()

Marca con un aspa los servicios con que cuentas en casa

Personal de servicio ( ) Lavadora ( ) Teléfono fijo ( ) Cable ( ) Internet ( )

Refrigeradora ( ) Cocina ( ) Nro. de baños ()

Colegio de procedencia

Club social o recreacional

Motivos de tu pertenencia al grupo

(Ordénalos con número según tu preferencia)

( ) Porque mi amigo (a) asistía y me invitó

( ) Porque quería hacer algo por los demás

( ) Porque quería sentirme útil

( ) Porque pienso que hay que realizar alguna actividad social o de liderazgo en la universidad, más allá de las cosas académicas de estudio

\section{Participación en el grupo}

( ) Frecuente 
( ) No tan frecuente

( ) Ocasional

\section{$\underline{\text { Instrucciones }}$}

- El objetivo de esta encuesta es conocer las motivaciones que tienes para pertenecer al grupo de organización, liderazgo, reflexión, ayuda social, voluntariado u otro similar que integras en la UNALM.

- Por favor, expresa tus opiniones y sentimientos acerca de las afirmaciones que siguen.

- Recuerda que no hay respuesta correcta ni incorrecta.

- Procura que tu experiencia personal decida tu respuesta real, más que tu respuesta ideal.

- Lee cuidadosamente cada frase y asegúrate de contestar a todas.

- Para cada frase necesitamos que respondas de acuerdo a una escala del 1 al 5 donde 1 es "Totalmente en desacuerdo" y 5 en "Totalmente de acuerdo".

\begin{tabular}{|l|c|c|c|c|c|c|}
\hline \multicolumn{1}{|c|}{ FRASES } & TA & & & & TD & $\begin{array}{c}\text { NS } \\
\text { NO }\end{array}$ \\
\hline $\begin{array}{l}\text { Ante situaciones en las que estoy expuesto, suelo mostrarme } \\
\text { más extrovertido y valiente, que introvertido y temeroso. }\end{array}$ & 5 & 4 & 3 & 2 & 1 & 99 \\
\hline $\begin{array}{l}\text { He tenido padres que me han expuesto a situaciones } \\
\text { de aprendizaje antes que haberme sobreprotegido y } \\
\text { encerrado en una burbuja, aislándome de mi entorno de } \\
\text { socialización. }\end{array}$ & 5 & 4 & 3 & 2 & 1 & 99 \\
\hline $\begin{array}{l}\text { En el periodo de la infancia he recibido fundamentalmente } \\
\text { mensajes de felicitación, por los logros, más que indiferencias } \\
\text { por las pequeñas metas alcanzadas. }\end{array}$ & 5 & 4 & 3 & 2 & 1 & 99 \\
\hline $\begin{array}{l}\text { Durante la etapa de mi nińez, disfrute mis veranos y tiempos } \\
\text { libres en actividades recreacionales, artísticas y deportivas, } \\
\text { antes que quedarme en casa desocupado y al cuidado de } \\
\text { un adulto. }\end{array}$ & 5 & 4 & 3 & 2 & 1 & 99 \\
\hline $\begin{array}{l}\text { Frente a los acontecimientos dolorosos, suelo reaccionar } \\
\text { más alegre y eufórico que triste y depresivo. }\end{array}$ & 5 & 4 & 3 & 2 & 1 & 99 \\
\hline $\begin{array}{l}\text { Los acontecimientos dolorosos han sido asumidos por mis } \\
\text { padres de manera equilibrada y hasta asertiva, antes que } \\
\text { haberles ocasionado tristeza y depresión. }\end{array}$ & 5 & 4 & 3 & 2 & 1 & 99 \\
\hline $\begin{array}{l}\text { A lo largo de mi infancia, pubertad y adolescencia, he oído } \\
\text { frases motivadoras y afirmativas frente a las habilidades } \\
\text { que iba adquiriendo más que mensajes de desconfianza y } \\
\text { descrédito. }\end{array}$ & 5 & 4 & 3 & 2 & 1 & 99 \\
\hline $\begin{array}{l}\text { Frecuentemente, disfrutaba de paseos familiares en días } \\
\text { libres o vacaciones, antes que quedarme en casa sin ninguna } \\
\text { actividad programada. }\end{array}$ & 5 & 4 & 3 & 2 & 1 & 99 \\
\hline $\begin{array}{l}\text { Yo soy una persona que cuando debe afrontar dificultades es } \\
\text { arriesgada y expresiva más que reservada y reprimida. }\end{array}$ & 5 & 4 & 3 & 2 & 1 & 99 \\
\hline $\begin{array}{l}\text { Mi infancia y pubertad la he pasado aprendiendo de mis } \\
\text { padres la expresión de los afectos y emociones, más que el } \\
\text { control represivo de los mismos. }\end{array}$ & 5 & 4 & 3 & 2 & 1 & 99 \\
\hline
\end{tabular}




\begin{tabular}{|c|c|c|c|c|c|c|}
\hline $\begin{array}{l}\text { Mi cuerpo ha registrado la caricia y el contacto físico como } \\
\text { signo de afecto y acercamiento más que el abuso físico como } \\
\text { signo de aprovechamiento e invasión de la intimidad. }\end{array}$ & 5 & 4 & 3 & 2 & 1 & 99 \\
\hline $\begin{array}{l}\text { El ambiente en que crecí estuvo caracterizado por el } \\
\text { encuentro y la comunicación entre los miembros de mi } \\
\text { familia, antes que por el desinterés y aislamiento de cada } \\
\text { uno en sus propias actividades. }\end{array}$ & 5 & 4 & 3 & 2 & 1 & 99 \\
\hline $\begin{array}{l}\text { Soy alguien más dado (a) a la acción y el compromiso, antes } \\
\text { que a la honda experimentación de una pasión o sueño. }\end{array}$ & 5 & 4 & 3 & 2 & 1 & 99 \\
\hline $\begin{array}{l}\text { Mis padres se han entregado normalmente a la construcción } \\
\text { de un gran sueńo o ideal, antes que al ejercicio activo de } \\
\text { compromisos por la realidad. }\end{array}$ & 5 & 4 & 3 & 2 & 1 & 99 \\
\hline $\begin{array}{l}\text { En la infancia y pubertad ha predominado la corrección } \\
\text { verbal ante mis errores, más que el insulto hiriente o el } \\
\text { castigo físico. }\end{array}$ & 5 & 4 & 3 & 2 & 1 & 99 \\
\hline $\begin{array}{l}\text { Siempre sentí que el espacio en que vivía era seguro y que } \\
\text { aunque mis padres no estuvieran por sus compromisos } \\
\text { importantes, nosotros nos quedábamos bajo al cuidado } \\
\text { de una persona de confianza, más que habernos sentido } \\
\text { abandonados en un espacio inseguro. }\end{array}$ & 5 & 4 & 3 & 2 & 1 & 99 \\
\hline $\begin{array}{l}\text { Ante situaciones en las que me siento amenazado, suelo } \\
\text { resolver las cosas con entusiasmo y optimismo más que } \\
\text { dramatizando o exagerando mi vivencia pesimistamente. }\end{array}$ & 5 & 4 & 3 & 2 & 1 & 99 \\
\hline $\begin{array}{l}\mathrm{He} \text { tenido padres optimistas frente a las situaciones } \\
\text { dramáticas que la vida conlleva, antes que exagerados o } \\
\text { pesimistas que dramatizaban sobre el drama. }\end{array}$ & 5 & 4 & 3 & 2 & 1 & 99 \\
\hline $\begin{array}{l}\text { Durante mi infancia y adolescencia, he escuchado sobre } \\
\text { todo frases de amabilidad y cortesía antes que de agresión } \\
\text { y grosería. }\end{array}$ & 5 & 4 & 3 & 2 & 1 & 99 \\
\hline $\begin{array}{l}\text { Durante mi infancia y pubertad, tuve la oportunidad de } \\
\text { intercambiar los espacios con personas que no pertenecían a } \\
\text { mi familia cercana, aprendiendo de los que eran diferentes } \\
\text { con sus propios intereses o costumbres, más que cerrar el } \\
\text { círculo de relaciones únicamente con los que eran iguales } \\
\text { o pares a mí. }\end{array}$ & 5 & 4 & 3 & 2 & 1 & 99 \\
\hline $\begin{array}{l}\text { Soy una persona que se muestra normalmente reflexiva } \\
\text { y consciente de lo que vive, más que vivir irreflexiva e } \\
\text { inconscientemente. }\end{array}$ & 5 & 4 & 3 & 2 & 1 & 99 \\
\hline $\begin{array}{l}\text { Mis padres son de los que enseñan a reflexionar acerca de } \\
\text { las cosas que experimentan los hijos antes que vivir siempre } \\
\text { inconsciente y apresuradamente. }\end{array}$ & 5 & 4 & 3 & 2 & 1 & 99 \\
\hline $\begin{array}{l}\text { Las frases que más he escuchado durante mi pubertad y } \\
\text { adolescencia han sido frases de comprensión y reconciliación } \\
\text { más que de condena y resentimiento. }\end{array}$ & 5 & 4 & 3 & 2 & 1 & 99 \\
\hline $\begin{array}{l}\text { Los ambientes para mi desarrollo han estado caracterizados } \\
\text { por la franca expresión de mis emociones, de mi autonomía } \\
\text { y de mi libertad, más que de la represión de mis emociones } \\
\text { y de las actividades sociales. }\end{array}$ & 5 & 4 & 3 & 2 & 1 & 99 \\
\hline
\end{tabular}




\begin{tabular}{|c|c|c|c|c|c|c|}
\hline $\begin{array}{l}\text { Son más las ocasiones en las que me muestro innovador e } \\
\text { informal que aquellas en las que soy conservador y formal. }\end{array}$ & 5 & 4 & 3 & 2 & 1 & 99 \\
\hline $\begin{array}{l}\text { He crecido con padres más innovadores e informales que } \\
\text { conservadores y formales. }\end{array}$ & 5 & 4 & 3 & 2 & 1 & 99 \\
\hline $\begin{array}{l}\text { En la infancia, pubertad y adolescencia, me han dicho frases } \\
\text { morales que me hacían sentir que yo era una persona buena, } \\
\text { más que frases que me hicieran sentir no tan bueno y hasta } \\
\text { malo. }\end{array}$ & 5 & 4 & 3 & 2 & 1 & 99 \\
\hline $\begin{array}{l}\text { En el barrio en el que crecí predominaba la colectividad } \\
\text { y el compromiso, más que el individualismo egoísta y } \\
\text { competitivo y el desinterés de unos hacia otros. }\end{array}$ & 5 & 4 & 3 & 2 & 1 & 99 \\
\hline $\begin{array}{l}\text { Cuando tengo que tomar decisiones importantes resuelvo } \\
\text { la situación con autonomía, en vez que sentir un impulso } \\
\text { natural de apoyarme en otros y depender de ellos. }\end{array}$ & 5 & 4 & 3 & 2 & 1 & 99 \\
\hline $\begin{array}{l}\text { Sobre todo, mis padres me han enseñado a ser autónomo } \\
\text { como ellos, más que apoyarse y depender de la aprobación, } \\
\text { consejo o decisión de otros. }\end{array}$ & 5 & 4 & 3 & 2 & 1 & 99 \\
\hline $\begin{array}{l}\text { Normalmente me daban mensajes que me hacían sentir } \\
\text { cercanía y atención a mis problemas y a mis procesos } \\
\text { personales, más que lejanía e indiferencia frente a ellos. }\end{array}$ & 5 & 4 & 3 & 2 & 1 & 99 \\
\hline $\begin{array}{l}\text { Fue una constante en mi desarrollo conocer ambientes y } \\
\text { realidades socioeconómicas distintas a las mías, frente a las } \\
\text { cuales aprendiera y me sensibilizara en brindar una ayuda, } \\
\text { antes que haber crecido en ambientes en los que se obviara } \\
\text { este tipo de exposición a situaciones difíciles. }\end{array}$ & 5 & 4 & 3 & 2 & 1 & 99 \\
\hline $\begin{array}{l}\text { Estoy más caracterizado por la ecuanimidad que por el } \\
\text { comportamiento obsesivo. }\end{array}$ & 5 & 4 & 3 & 2 & 1 & 99 \\
\hline $\begin{array}{l}\text { Mis padres son emocionalmente personas ecuánimes y } \\
\text { saludables, poco caracterizados por comportamientos } \\
\text { obsesivos. }\end{array}$ & 5 & 4 & 3 & 2 & 1 & 99 \\
\hline $\begin{array}{l}\text { Las frases que más he escuchado han sido normalmente } \\
\text { frases positivas antes situaciones críticas, más que las frases } \\
\text { negativas y poco estimulantes. }\end{array}$ & 5 & 4 & 3 & 2 & 1 & 99 \\
\hline $\begin{array}{l}\text { Mi casa era una casa de libertad y de relativa permisividad, } \\
\text { antes que una casa en las que habían ciertas normas y } \\
\text { prohibiciones para los niños y donde se respirara un clima } \\
\text { de formalismo y respeto a los mayores. }\end{array}$ & 5 & 4 & 3 & 2 & 1 & 99 \\
\hline $\begin{array}{l}\text { Soy de los que toman decisiones resueltamente, más que de } \\
\text { los que complica. }\end{array}$ & 5 & 4 & 3 & 2 & 1 & 99 \\
\hline $\begin{array}{l}\text { Mis padres toman las decisiones resueltamente y no se } \\
\text { complican tanto con las consecuencias o comprensión de la } \\
\text { complejidad de un problema que tienen al frente. }\end{array}$ & 5 & 4 & 3 & 2 & 1 & 99 \\
\hline $\begin{array}{l}\text { Durante mi infancia y pubertad, he escuchado frases que } \\
\text { me relajaban y calmaban frente a situaciones de angustia } \\
\text { antes que recibir mensajes que me estresaran y llenaran de } \\
\text { angustia y apresuramiento. }\end{array}$ & 5 & 4 & 3 & 2 & 1 & 99 \\
\hline
\end{tabular}

